\title{
Bone mineral density in cystic fibrosis patients using low-dose chest computed tomography: a pilot study
}

\author{
To the Editor:
}

Bone mineral density is usually normal in children with a good nutritional status and preserved lung function, and commonly reduced in adolescents and adults with cystic fibrosis $[1,2]$. However, several studies have documented the prevalence of low bone mineral density in children [3, 4], with $28-47 \%$ of them with osteopenia and 20-34\% with osteoporosis. STAHL et al. [5] observed that approximately $42 \%$ of cystic fibrosis patients present some type of bone fracture up to 25 years.

Early detection of altered bone mineral density is important for monitoring and assessing bone health status. Dual energy X-ray absorptiometry (DXA) is currently the gold standard method for measuring bone mineral density [5]. However, it is considered limited in young individuals with cystic fibrosis [6]. Other methods are used to evaluate bone mineral density, including central and peripheral quantitative computed tomography (QCT), ultrasound, radiography and magnetic resonance imaging [7].

In addition, measures such as Hounsfield score (HU), a standardised computed tomography (CT) attenuation coefficient, can provide information necessary for the diagnosis of bone mineral density reduction, with no additional patient costs and no extra radiation exposure in examinations obtained for clinical purposes [8,9]. SCHreiber et al. [9] in a cross-sectional study of adults found a significant correlation between Hounsfield score and bone mineral density $(\mathrm{r}=0.44 ; \mathrm{p}<0.0001)$. However, we could not find previous studies using chest CT to assess bone mineral density in patients with cystic fibrosis. Considering the elevated number of examinations and tests that cystic fibrosis patients undergo, the benefit of assessing lung and bone health using one test may be even higher.

The purpose of this study was to determine the correlation between Hounsfield score at the thoracic vertebrae and DXA at the lumbar vertebrae in children and adolescents with cystic fibrosis.

This was a retrospective cross-sectional study. All cystic fibrosis patients aged between 8 and 19 years, with chest CT and DXA scans, assisted in the outpatient cystic fibrosis clinic of Hospital São Lucas, were included.

The diagnosis of patients with cystic fibrosis was confirmed according to the Cystic Fibrosis Foundation Consensus Report [10]. Demographic, clinic and nutritional data of subjects were collected from their electronic records. We collected data on genetic mutation, bacterial colonisation and lung function. Z-scores of body mass index (BMI) by age and height by age were calculated, and classified according to the World Health Organization $[11,12]$.

Bone mineral density was evaluated by thoracic CT (measured by Hounsfield score) and lumbar DXA (measured by $\mathrm{g} \cdot \mathrm{cm}^{-2}$ ). Thoracic CT was performed using a scanner CT 16 multislice (LightSpeed VCT; GE Healthcare, Milwaukee, WI, USA), according to the protocol with collimation of the $1.25-\mathrm{mm}$ reconstruction Gantry rotation of $0.5 \mathrm{~s}, 80 \mathrm{kV}$ and $30 \mathrm{mAs}$ [13]. We measured the Hounsfield score in three consecutive thoracic vertebrae (T10, T11, T12) using the region of interest placed at the centre of the vertebrae, avoiding cortical bone, large vessels and lesions. The mean Hounsfield score was calculated by sum of three measures. All patients were instructed prior to examination to maintain normal breathing.

@ERSpublications

Bone mineral density assessed using a low-dose chest computed tomography in cystic fibrosis presented a strong correlation with DXA. For CF patients, there is an additional possible benefit of assessing lung and bone health using only one method. http://bit.ly/2vsCYrQ

Cite this article as: Feijó Andrade RG, Forte GC, Hochhegger B, et al. Bone mineral density in cystic fibrosis patients using low-dose chest computed tomography: a pilot study. Eur Respir J 2019; 53: 1900066 [https://doi.org/10.1183/13993003.00066-2019]. 
DXA scans were performed on a Hologic Discovery Wi densitometer (Hologic Inc., Waltham, MA, USA). Information from the DXA scans, including z-scores and bone mineral density (measured in $\mathrm{g} \cdot \mathrm{cm}^{-2}$ ), were obtained for the first through fourth lumbar vertebrae [14]. The bone mineral density was classified by International Society for Clinical Densitometry, considering z-score $\leqslant 2$ as low bone mineral density. These examinations are performed routinely (in annual check-up) in patients with cystic fibrosis, at ages between 8 and 19 years.

All statistical analyses were performed using SPSS (version 17.0; IBM Corporation, Armonk, NY, USA). Continuous variables were presented as mean $\pm \mathrm{SD}$ or median (interquartile range) for asymmetric distributions. Categorical variables were presented as frequencies and percentages. Pearson's correlation coefficient was calculated to evaluate the association between bone mineral density measured by thoracic CT and DXA. p-values $<0.05$ were considered significant. The study was approved by the Ethics Committee at the Pontifícia Universidade Católica do Rio Grande do Sul, Brazil (CAAE: 49692115.7.0000.5336).

A total of 18 children and adolescents, with mean age $16.1 \pm 3.4$ years, were evaluated. There was a predominance of males $(66.7 \%)$ and $15(83.3 \%)$ participants were Caucasians. Three (16.7\%) patients were homozygous for F508del, while nine (50\%) were heterozygous for F508del; the remaining six (33.3\%) patients carry other CFTR mutations. Pseudomonas aeruginosa was found in six (33.3\%) patients. Mean forced expiratory volume in $1 \mathrm{~s}$ was $73.6 \pm 32.5 \%$ pred. The median BMI z-score was 0.03 ( -0.88 to 0.81 ) and $15(83.4 \%)$ patients were classified as normal weight.

The median of bone mineral density z-score by DXA was $0.65(-1.60$ to 0.20$)$ and the mean of thoracic CT was 229.2 \pm 30.6 HU. 15 (83.3\%) patients were diagnosed as normal and three (16.7\%), as low bone mineral density.

A strong positive correlation was observed between bone mineral density measured by thoracic CT and DXA $(\mathrm{r}=0.740 ; \mathrm{p}<0.001)$ (figure 1).

We found a strong positive correlation between bone mineral density measured by thoracic CT and DXA in children and adolescent with cystic fibrosis. In addition, our study results may provide additional early information on bone disease in cystic fibrosis patients.

Chest CT is a frequently used to evaluate progression of cystic fibrosis lung disease. Hounsfield score obtained from CT scans to assess the bone mineral density was described by SCHREIBER et al. [9] in 25 patients with mean age of 71.3 years. The results showed a significant correlation between Hounsfield score and DXA, and they concluded that CT data may be useful for the diagnosis of osteoporosis.

Many studies have evaluated bone mineral density by DXA or peripheral QCT $[6,15,16]$. However, these methods result in an additional cost. According to international guidelines, DXA scan should first be performed from about 8 to 10 years [2]. It is a bidimensional method that does not differentiate the cortical and trabecular bone. In addition, peripheral QCT is not widely available, technically demanding and, consequently, it not commonly used in clinical practice.

This study has some methodological limitations like a small sample size, retrospective cross-sectional design, single reference centre and DXA as reference standard. The fact that only three subjects had low bone mineral density is a further limitation. In addition, there is no paediatric reference database to determine z-scores when evaluating bone mineral density by CT of the thoracic vertebrae. Establishing normative data would be a critical step in developing the utility of chest CT to screen for decreased bone

FIGURE 1 Scatter graph of bone mineral density measured by thoracic computed tomography (CT) in Hounsfield units (HU) and by dual energy X-ray absorptiometry (DXA) in $\mathrm{g} \cdot \mathrm{cm}^{-2}$ in children and adolescents with cystic fibrosis.

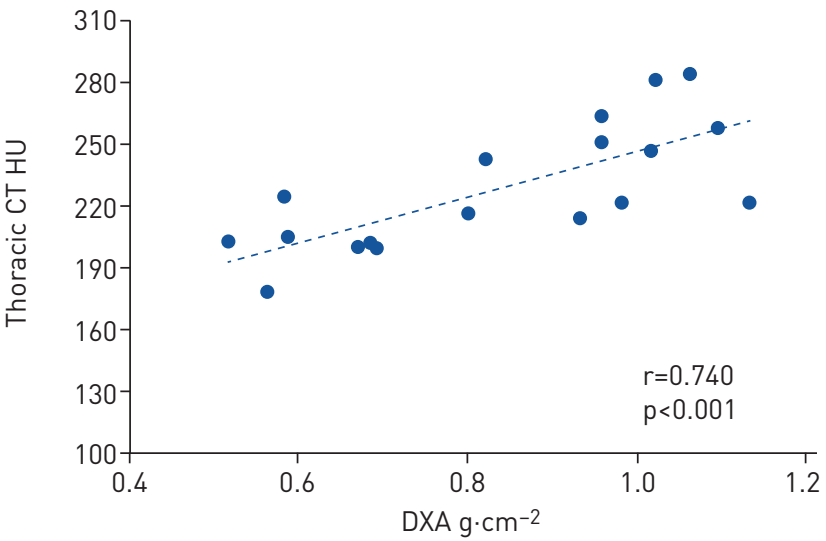


density in children. In spite of these concerns, this is the first study to evaluate the correlation between Hounsfield score and DXA in children with cystic fibrosis. Bone health status measured by thoracic CT provides many advantages, such as differentiating the cortical and trabecular bone; it is also possible to evaluate fractures and their complications. It is a simple method and widely used by radiologists, performed without additional cost and in a clinical setting. For cystic fibrosis patients, there is the additional possible benefit of assessing lung and bone health using only one method.

In conclusion, the present study showed a strong positive correlation between thoracic CT and lumbar DXA to evaluate bone health in children and adolescents with cystic fibrosis, suggesting the possibility of early diagnosis, and thereby enabling the development of new strategies to prevent and treat bone disease. Further studies with larger sample sizes are necessary to confirm the usefulness of chest CT to assess bone health in cystic fibrosis patients.

Rubens Gabriel Feijó Andrade $\oplus^{1}$, Gabriele Carra Forte ${ }^{1}$, Bruno Hochhegger ${ }^{2}$ and Leonardo Araujo Pinto

${ }^{1}$ Programa de Pós-graduação em Pediatria e Saúde da Criança, Pontifícia Universidade Católica do Rio Grande do Sul, Porto Alegre, Brazil. ${ }^{2}$ Programa de Pós-graduação em Medicina e Ciências da Saúde, Pontifícia Universidade Católica do Rio Grande do Sul, Porto Alegre, Brazil.

Correspondence: Rubens Gabriel Feijó Andrade, Pontifícia Universidade Católica do Rio Grande do Sul (PUCRS), Avenida Ipiranga 6690, 1st floor, Porto Alegre 90610-000, RS, Brazil. E-mail: rubensfeijoandrade@gmail.com

Received: Jan 112019 | Accepted after revision: March 132019

Conflict of interest: None declared.

Support statement: Fundação de Amparo à Pesquisa do Rio Grande do Sul (FAPERGS), the National Research Council of Brazil $(\mathrm{CNPq})$ and Coordenação de Aperfeiçoamento de Pessoal de Nível Superior CAPES.

\section{References}

1 Smith N, Lim A, Yap M, et al. Bone mineral density is related to lung function outcomes in young people with cystic fibrosis-a retrospective study. Pediatr Pulmonol 2017; 52: 1558-1564.

2 Castellani C, Cuppens $\mathrm{H}$, Macek M, et al. Consensus on the use and interpretation of cystic fibrosis mutation analysis in clinical practice. J Cyst Fibros 2008; 7: 179-196.

3 Gronowitz E, Garemo M, Lindblad A, et al. Decreased bone mineral density in normal-growing patients with cystic fibrosis. Acta Paediatr 2003; 92: 688-693.

4 Sermet-Gaudelus I, Castanet M, Retsch-Bogart G, et al. Update on cystic fibrosis-related bone disease: a special focus on children. Paediatr Respir Rev 2009; 10: 134-142.

5 Stahl M, Holfelder C, Kneppo C, et al. Multiple prevalent fractures in relation to macroscopic bone architecture in patients with cystic fibrosis. J Cyst Fibros 2018; 17: 114-120.

6 Brookes DSK, Briody JN, Munns CF, et al. Cystic fibrosis-related bone disease in children: examination of peripheral quantitative computed tomography (pQCT) data. J Cyst Fibros 2015; 14: 668-677.

7 Ferris H, Twomey M, Moloney F, et al. Computed tomography dose optimisation in cystic fibrosis: a review. World J Radiol 2016; 8: 331.

8 Schreiber JJ, Anderson PA, Hsu WK. Use of computed tomography for assessing bone mineral density. Neurosurg Focus 2014; 37: E4.

9 Schreiber JJ, Anderson PA, Rosas HG, et al. Hounsfield units for assessing bone mineral density and strength: a tool for osteoporosis management. J Bone Joint Surg Am 2011; 93: 1057-1063.

10 Farrell PM, Rosenstein BJ, White TB, et al. Guidelines for diagnosis of cystic fibrosis in newborns through older adults: Cystic Fibrosis Foundation Consensus Report. J Pediatr 2008; 153: S4-S14.

11 De Onis M, Onyango AW, Borghi E, et al. Development of a WHO growth reference for school-aged children and adolescents. Bull World Health Organ 2007; 85: 660-667.

12 Turck D, Braegger CP, Colombo C, et al. ESPEN-ESPGHAN-ECFS guidelines on nutrition care for infants, children, and adults with cystic fibrosis. Clin Nutr 2016; 35: 557-577.

13 de Jong PA, Tiddens HAWM. Cystic fibrosis specific computed tomography scoring. Proc Am Thorac Soc 2007; 4: 338-342.

14 Schousboe JT, Shepherd JA, Bilezikian JP, et al. Executive summary of the 2013 International Society for Clinical Densitometry Position Development Conference on bone densitometry. J Clin Densitom 2013; 16: 455-466.

15 Pezzuti IL, Kakehasi AM, Filgueiras MT, et al. Imaging methods for bone mass evaluation during childhood and adolescence: an update. J Pediatr Endocrinol Metab 2017; 30: 485-497.

16 Bai W, Binkley TL, Wallace JW, et al. Peripheral quantitative computed tomography (pQCT) bone measurements in children with cystic fibrosis. Pediatr Pulmonol 2016; 51: 28-33. 